\title{
Surface-enhanced Raman fiberoptic sensors for remote monitoring
}

\author{
D. L. Stokes, J.P. Alarie, T. Vo-Dinh*
}

Oak Ridge National Laboratory, Health Sciences Research Division, Advanced Monitoring Development Group, Oak Ridge, TN 37831-6101, USA

\begin{abstract}
A new sensor design for remote surface-enhanced Raman scattering (SERS) measurements has been developed for environmental applications. The design features the modification of an optical fiber using layers of alumina microparticles and silver coatings for inducing the SERS effect at the sensing probe. A single fiber carries both the laser excitation and the SERS signal radiation, keeping optical parameters at the remote tip simple and consistent. The small tip size achievable with this configuration also demonstrates potential of this new design as a microsensor for in-situ measurement in microenvironments. Details of sensor tip fabrication and optical system design are described. SERS spectra of aqueous environmental samples acquired in-situ using the SERS sensor are also presented to illustrate the effectiveness of the SERS sensor.
\end{abstract}

\section{INTRODUCTION}

Raman spectroscopy has the potential of being a valuable tool in environmental analysis due to recent advances in the surface-enhanced Raman scattering SERS technology. The very narrow, highly-resolved vibrational bands associated with the Raman scattering process not only yield structural information about analyte molecules, but also offer a spectral selectivity which is comparable to that of infrared (IR) absorption spectroscopy. The spectral selectivity can enable the analysis of complex mixtures without excessive sample pretreatment, thus making it particularly useful for in-situ environmental sampling. However, the inherently low cross-section of normal Raman scattering has been a severe limiting factor for its use as an analytical tool. In 1974, Fleischmann and coworkers discovered a giant enhancement in the Raman signal from compounds adsorbed on or near roughened silver electrodes. 1 This enhancement factor has been confirmed by Van Duyne et al. to be as high as $10^{7} .2$ These discoveries have initiated great interest in SERS as a sensitive analytical technology. In this work, we introduce a SERS-based optical fiber sensor which exhibits the potential for remote analysis.

Increasing use of the SERS technology has involved extensive efforts in the development of theories and the substrate technology utilizing the giant Raman signal enhancement. The theory for SERS can generally be described in terms of a chemical model and an electromagnetic model, both requiring a roughened surface. The short-range chemical model involves the formation of an "adatom" via adsorption of the analyte with the roughened metal surface and has been described as being dependant on the atomic-scale roughness of the surface.3,4 The long-range electromagnetic model involves the generation of intense fields concentrated at protrusions in the roughened surface. These fields are induced by the oscillation of conduction electrons (surface plasmons) in the metal surface. . $^{-7}$ The electromagnetic enhancement is dependant on large-scale roughness. ${ }^{8-10}$ Based on these theories, several media have been developed to utilize the SERS effect, including silver-coated quartz posts, ${ }^{11}$ metal island films 12,13 and metalcoated microspheres. 14,15

SERS-based optical fiber sensors developed thus far vary widely in design. For example, sensors based on thiol-

* Corresponding Author

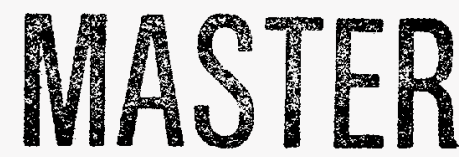

DISTABUTION OF THIS DOCUMENT IS UNLIMITED WWV

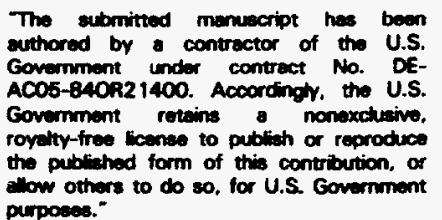




\section{DISCLAIMER}

Portions of this document may be illegible in electronic image products. Images are produced from the best available original document. 
modified metal surfaces have been reported for the selective adsorption of metal ions and aromatic compounds. 16,17 Sensors based on electrochemical adsorption have also been reported.18,19 Some designs have enabled SERS detection with fibers as long as $250 \mathrm{~m} .{ }^{20}$ In our laboratory, we have developed optical fiber sensors based on silvercoated microspheres or microparticles. The original design coupled separate excitation and optical fibers to a flat, detachable, silver-coated microsphere-based substrate. 21 The substrates used in this design have been successfully used for the detection of chemicals of environmental concern, including polycyclic aromatic compounds, 14 organophosphorus compounds, ${ }^{22}$ chlorinated pesticides, ${ }^{23}$ fungicides, 24 and other growth inhibitors. ${ }^{25}$ In addition we have used these substrates to investigate cosmetic and food products, ${ }^{26}$ DNA products, ${ }^{27}$ and gene probes. ${ }^{28}$ We have also been able to couple this probe to a red-enhanced intensified charge-coupled device (RE-ICCD) for rapid remote sensing. 29

In this work, we describe a new single-fiber design in which the fiber itself is modified for SERS activity. There is no need for a separate substrate. The tip of the single fiber is coated with layers of alumina microspheres and then coated with silver. A holographic beam splitter used in the optical system allows the use of the single fiber for the transmission of both laser excitation and SERS signal radiation. With this new system, the lack of complicated optical components at the sensor tip provides the potential for improved signal reproducibility. The size of this new sensor is also much smaller than the original design, making in-situ measurements in microenvironments feasible. By coupling this probe to an RE-ICCD detection system, we have been able to rapidly acquire data, in-situ, on aqueous environmental samples.

\section{EXPERIMENTAL}

\subsection{Sensor Tip Fabrication}

The SERS sensor tips were made from a $600-\mu \mathrm{m}$ diameter silica optical fiber (Fiberguide Industries) which was cleaved into 8-cm sections. The ends of these sections were stripped of the jacket and cladding, cleaned and polished. The stripping exposed $1-\mathrm{cm}$ lengths of silica fiber from each end. Cleaning included rinsing with dilute nitric acid, water and ethanol. Polishing was performed with alumina polishing sheets $(0.5-\mu \mathrm{m}$ grain). The newly polished fibers were then dipped (one end only) in a 5\% aqueous suspension of alumina (Baikowski International, Charlotte, NC) for about three seconds. The fibers were then allowed to dry and placed in a vacuum evaporation system (Cooke Vacuum Products, Model CV-301) for application of the silver coating. Silver deposition was performed at a rate of approximately $1.5 \AA / s$ at a pressure of $2 \times 10^{-6}$ Torr. The thickness of the silver coating was $1000 \AA$.

\subsection{Instrumentation}

The optical system used for this work is illustrated in Figure 1. The excitation source was a helium-neon laser (Spectra Physics, Model 106-1). The 632.8-nm line was spectrally purified with a narrow bandpass filter (FWHM = $3.0 \pm 0.5 \mathrm{~nm}$ )(Corion, Model P3-633-A-X516). The beam intensity emerging from the bandpass filter was approximately $3 \mathrm{~mW}$. A holographic beam-splitter (Kaiser, Model HB-633-1.0) reflected the 632.8-nm radiation into a 10x objective lens, which focused the radiation onto the SERS-active optical fiber tip. The Raman scattering signal returning from the SERS-active tip and emerging from the sensor optical fiber was collected and collimated by the same objective lens. The Raman-shifted radiation was transmitted through the holographic beam splitter towards a second optical fiber, while the 632.8-nm radiation was reflected back towards the laser. A holographic notch filter (Physical Optics Corporation) was used to reject any Rayleigh-scattered radiation that was not diverted from the optical axis by the beam splitter. An f/l lens was used to focus the SERS signal onto the second optical fiber which transmitted the SERS signal to a spectrograph (Instruments SA, Model HR-320), which was equipped with a $600-\mathrm{gr} / \mathrm{mm}$ grating. Reciprocal linear dispersion was $50 \mathrm{~nm} / \mathrm{mm}$. All measurements were performed with an entrance slit width of $30 \mu \mathrm{m}$, resulting in a resolution of approximately $3.3 \mathrm{~cm}^{-1}$ at $675 \mathrm{~nm}$. Multichannel 
detection was performed with a red-enhanced intensified charge-coupled device (RE-ICCD)(Princeton Instruments, Model RE/ICCD-576S). This detector was thermoelectrically-cooled to $-34^{\circ} \mathrm{C}$. A software package designed by the Princeton Instruments specifically for this detector was used for all data acquisition and processing.

\section{RESULTS AND DISCUSSIONS}

\subsection{Dry probe detection.}

Figure 2a illustrates the SERS spectrum of 2,4-dinitrophenol (DNP) acquired with the new SERS sensor. 2,4dinitrophenol was selected since this compound is related to explosive contaminants in aqueous environments. The spectrum was acquired by first dipping the tip into an aqueous solution of the DNP at $2.2 \times 10^{-3} \mathrm{M}$ concentration. The solution was contained in a micro centrifuge tube. After submerging the sensor tip in the solution for approximately 1 minute, the sensor was removed and allowed to dry. The spectrum was also acquired using an accumulation of 10 RE-ICCD exposures of $10 \mathrm{~s}$ each. A blank spectrum of the dry sensor probe tip acquired before submersion in the DNP is illustrated by Figure $2 \mathrm{~b}$. All experimental parameters were constant for both spectra except for the RE-ICCD gain, which was higher for the blank. The higher intensity level demonstrated by the blank is entirely due to the higher detector gain level, which was selected to elucidate any possible structure exhibited by the blank. This blank spectrum shows no significant SERS bands in the spectral region of interest.

\subsection{Submerged probe detection.}

Figure 3a illustrates the SERS spectrum of cresyl fast violet (CFV) acquired from a $1.8 \times 10^{-6} \mathrm{M}$ aqueous solution. The CFV compound is a good tracer compound to monitor underground water migration. In this case the SERS sensor was dipped in the analyte solution and left submerged for the duration of the measurement. The spectrum was acquired as the accumulation of 5 exposures of $5 \mathrm{~s}$ each. The solution was contained in a microcentrifuge tube and was not stirred. We have observed that the SERS signals from solutions measured in-situ vary with time. This particular spectrum was taken after 35 minutes of submersion. A blank spectrum of the sensor submerged in pure water is also illustrated by Figure $3 \mathrm{~b}$. This spectrum was taken before exposure to an analyte solution. The high intensity level exhibited by the blank spectrum is due to the higher detector gain level of the detector which was again selected for the investigation of minor structures in the blank spectrum. Otherwise, experimental parameters were held constant for the two spectra. As can be seen by the figure, however, no significant structure is exhibited by the blank spectrum for the spectral region of interest.

\subsection{Ground Water Sample}

Figure 4 demonstrates the spectrum of a ground water sample containing $500 \mathrm{ppb}\left(1.5 \times 10^{-6} \mathrm{M}\right) \mathrm{CFV}$. The ground water was obtained from an industrial waste burial site. In this case the fiber sensor tip was left submerged in the environmental sample for the duration of the measurement. The spectrum, representing an accumulation of 5 exposures of $5 \mathrm{~s}$ each, was taken 30 minutes subsequent to sensor submersion. This example demonstrates the potential of monitoring underground water migration with CFV using the new SERS sensor.

In conclusion, a new SERS-based sensor has been described for the remote analysis of environmental samples. It consists of a single optical fiber modified to induce the SERS effect. The fiber is coated with layers of alumina microspheres and silver and is, in this respect, very similar to the flat plate microsphere-based substrates introduced in earlier work. 15 Our work has illustrated that the sensor can be successfully used by either dipping the probe in a remote sample and then allowing the tip to dry after retraction from the sample before measurement, or by submerging the sensor tip in the sample and acquiring real-time spectra during submersion. This study demonstrates the feasibility of a SERS fiberoptic sensor for in-situ detection of chemicals for environmental applications. 


\section{Acknowledgments}

This work was sponsored by the U. S. Department of Energy (DOE) Office of Health and Environmental Research under Contract DE-AC05-84OR21400 with Lockheed Martin Energy Systems, Inc. The authors also acknowledge the support of the U. S. Department of Defense BMDO (DGAM4-0238).

\section{LITERATURE CITED}

1) Fleischmann, M. J.; Hendra, P. J.; MacQuillan, A. J. Chem. Phys. Lett. 1974, 26, 163-166.

2) Jeanmaire, D. J.; Van Duyne, R. P. J. Electroanal. Chem. Interfacial Electrochem. 1977, 84, 1-20.

3) Otto, A. ; Mrozek, I.; Grabhorn, H.; Akemann, W. J. J. Phys. Condens. Matter 1992, 4, 1143 1212.

4) Otto, A. J. Raman Spectrosc. 1991, 22, 743-752.

5) Gertsen, J.; Nitzan. A. J. Chem. Phys. 1980, 73, 3023-3037.

6) Kerker, M.; Wang, D. S.; Chew, H. Appl. Opt. 1980, 19, 4159-4174.

7) Ferrell, T. L. Phys, Rev. B: Condens. Matter 1982, 25, 2930-2932.

8) Schatz, G.C. Acc. Chem. Res. 1984, 17, 370-376.

9) Zeman, E. J.; Schatz, G. C. In Dynamics on Surfaces: The Jerusalem Symposia on Quantum Chemistry and Biochemistry; Pullman, B., Jortner, J., Gerber, B., Nitzan, A., Eds.; D. Reidel Publishing Co.: Dordrecht, Holland, 1984; Vol. 17, pp 413-424.

10) Zeman, E. J.; Schatz, G. C. J. Phys. Chem. 1987, 91, 634-643.

11) Vo-Dinh, T.; Meier, M; Wokaun, A. Anal. Chim. Acta 1986, 181, 139-148.

12) Jennings, C,; Avoca, R.; Hor, A.; Loufyt, R. O. Anal.Chem. 1984, 56, 2033-2035.

13) Berthod, A.; Laserna, J. J.; Winefordner, J. D. Appl. Spectrosc. 1987, 41, 1137-1141.

14) Vo-Dinh, T.; Hiromoto, M. Y. K.; Begun, G. M.; Moody, R. L. Anal. Chem. 1984, 56, 1667- 1670.

15) Bello, J. M.; Stokes, D. L.; Vo-Dinh, T. Appl. Spectrosc. 1989, 43, 1325-1330.

16) Heyns, J. B. B.; Sears, L. M.; Corcoran, R. C.; Carron, K. T. Anal. Chem. 1994, 66, 1572-1574.

17) Carron, K.; Peiterson, L.; Lewis, M. Environ. Sci. Technol. 1992, 26, 1950-1954.

18) Angel, S.; Archibald, D. Appl. Spectrosc. 1989, 43, 1097-2102.

19) Carrabba, M.; Edmonds, R.; Rauh, R. Anal. Chem. 1987, 59, 2559-2563.

20) Myrick, M. L. and Angel, S. M. Appl. Spectrosc. 1990, 44, 565-569.

21) Bello, J. M.; Vo-Dinh, T. Appl. Spectrosc. 1990, 44, 63-69.

22) Alak, A. M.; Vo-Dinh, T. Anal.Chem. 1987, 59, 2149-2153.

23) Alak, A. M.; Vo-Dinh, T. Anal.Chem. Acta 1988, 206, 333-337. 
24) Narayanan, V. A.; Begun, G. M.; Stokes, D. L.; Sutherland, W. S.; Vo-Dinh, T. J. Raman Spectrosc. 1992, 23, 281-286.

25) Narayanan, V. A.; Begun, G. M.; Bello, J. M.; Stokes, D. L.; Vo-Dinh, T. Analusis 1993, 21, 107-112.

26) Narayanan, V. A; Stokes, D. L.; Stump, N. A.; Begun, G. M.; Vo-Dinh, T. Polycyclic Aromatic Compounds 1993, 3, 137-146.

27) Helmenstine, A.; Uziel, M.; Vo-Dinh, T. J. Toxicol. and Env. Health 1993, 40, 195-202.

28) Vo-Dinh, T.; Houck, K.; Stokes, D. L. Anal. Chem. 1994, 66, 3379-3383.

29) Alarie, J. P.; Stokes, D. L.; Sutherland, W. S.; Edwards, A. C.; Vo-Dinh, T. Appl. Spectrosc. 1992, 46, 1608-1612.

\section{DISCLAIMER}

This report was prepared as an account of work sponsored by an agency of the United States Government. Neither the United States Government nor any agency thereof, nor any of their employees, makes any warranty, express or implied, or assumes any legal liability or responsibility for the accuracy, completeness, or usefulness of any information, apparatus, product, or process disclosed, or represents that its use would not infringe privately owned rights. Reference herein to any specific commercial product, process, or service by trade name, trademark, manufacturer, or otherwise does not necessarily constitute or imply its endorsement, recommendation, or favoring by the United States Government or any agency thereof. The views and opinions of authors expressed herein do not necessarily state or reflect those of the United States Government or any agency thereof. 


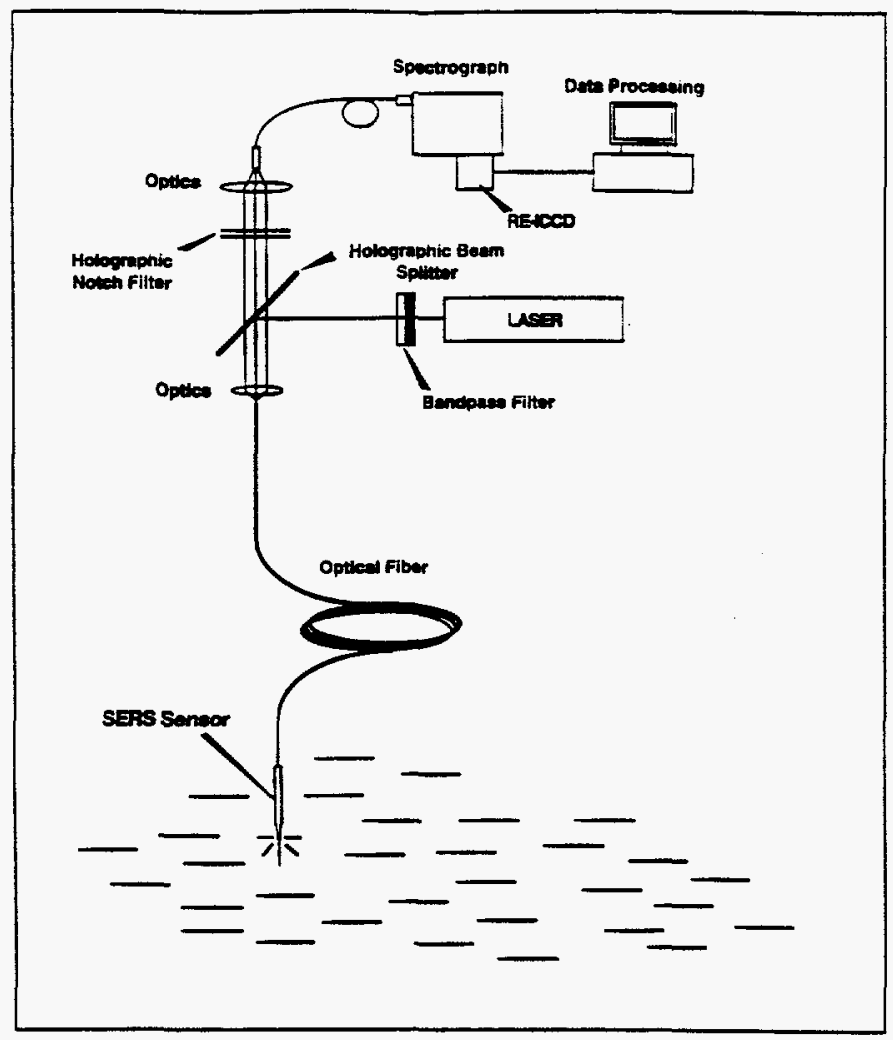

Eigure 1. Schematic representation of the SERS sensor system for remote analysis.

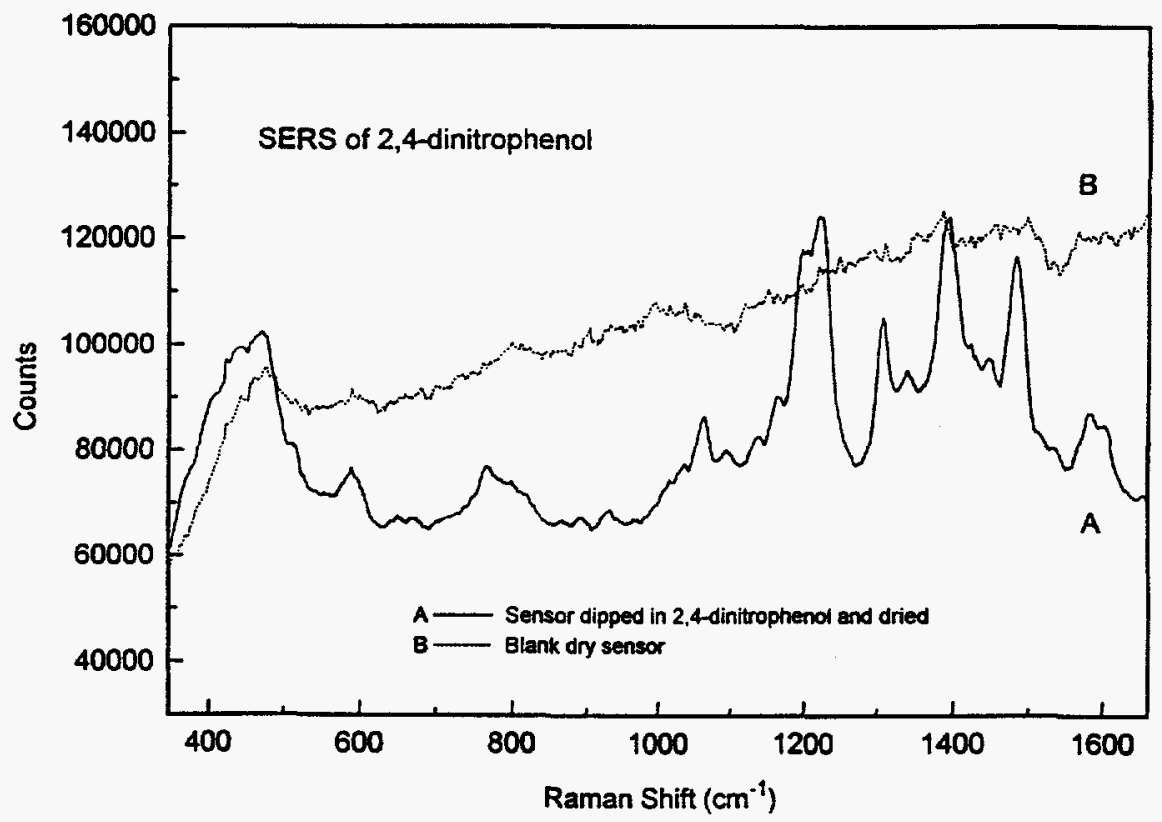

Eigure 2. a) SERS spectrum of dry sensor after submersion in an aqueous solution of $2.2 \times 10^{-3} \mathrm{M} 2,4-$ dinitrophenol, and b) blank spectrum of dry sensor prior to exposure to analyte. 


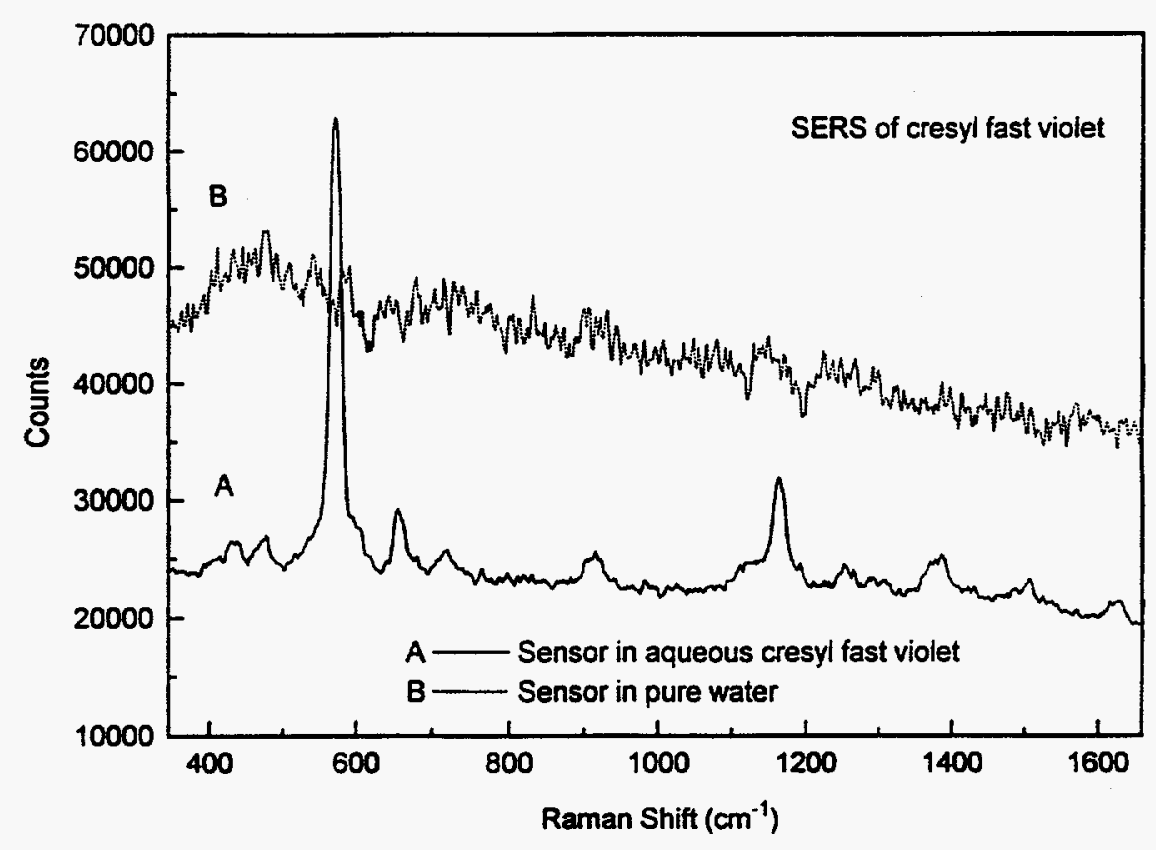

Figure 3 a) SERS spectrum acquired during sensor submersion in an aqueous solution of $1.8 \times 10^{-6} \mathrm{M}$ cresyl fast violet, and b) SERS spectrum acquired during sensor submersion in pure water.

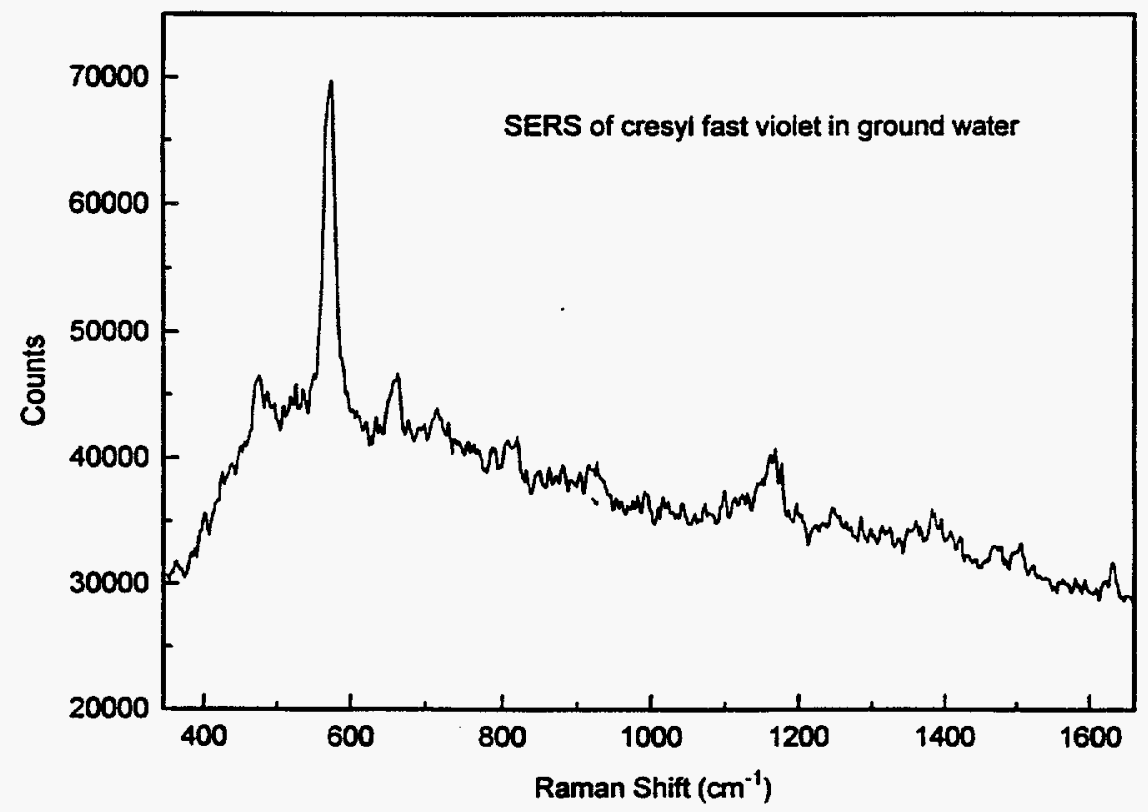

Figure 4. SERS spectrum of an industrial site ground water sample containing $1.5 \times 10^{-6} \mathrm{M}$ cresyl fast violet. 